\section{Peripheral neuropathy in leprosy: Clinical manifestations and disability in a Colombian national referral center}

\author{
Laura Trujillo-Ramirez, ${ }^{1}$ \\ María Alejandra Palacios-Ariza, ${ }^{2}$ \\ Ivan Pradilla, ${ }^{3}$ Luis Arturo Gamboa ${ }^{4}$ \\ ${ }^{1}$ Faculty of Medicine, Fundación \\ Universitaria Sanitas, Bogotá; ${ }^{2}$ Research \\ Unit, Fundación Universitaria Sanitas, \\ Bogotá; ${ }^{3}$ Department of Neurology, \\ Fundación Cardioinfantil, Bogotá; \\ ${ }^{4}$ University Hospital, Dermatologic \\ Center Federico Lleras Acosta, \\ Fundación Universitaria Sanitas, \\ Bogotá, Colombia
}

\begin{abstract}
To aim of the paper was to describe the neurological features of the physical examination in patients with Hansen's disease who were treated in Bogotá, Colombia. We carried out a descriptive study of all patients with a Hansen's disease diagnosis treated at a referral center between 2003-2018. There were 327 eligible electronic health records (EHRs) with a final sample of 282 subjects. Leprosy was most common in males $(57.45 \%)$, median age at the diagnosis was 54 years, and lesions of the lower limbs were more common $(75.1 \%)$. The median time from disease onset to consult was 12 months. Most of them were classified as having lepromatous leprosy (39.7\%). Pain over the median nerve trunk was the most common manifestation of disease $(28 \%)$, followed by pain over the radial trunk $(22 \%)$. Sensitive alterations were more common than motor ones. Specifically, the posterior tibial nerve was affected in nearly half of subjects. Dual impairment was more common in the ulnar nerve $(13.8 \%)$. Some disability was apparent in $23.8 \%$ of subjects; predominantly grade 1 disability. Findings regarding age, leprosy type, and the frequency of individual nerve compromise were consistent with reports from other countries. Nerve trunk thickening was infrequent, which might be a consequence of subjectiveness in the examination and sample differences in sex distribution, degree of disability and time since disease onset. The frequency of morbidity and disability found in this sample, though low when compared with other series, fails to meet public health goals, including those limiting disability in younger subjects.
\end{abstract}

\section{Introduction}

In the global update report published by the World Health Organization (WHO) for 2019, a total of 202,185 new cases of Hansen's disease were reported (25.9 per million inhabitants), of which 10,813 had grade 2 disability. ${ }^{1}$ This report showed that disease transmission persists and is a public health issue in some countries. Such is the case in Colombia, where detection rates have been reduced only minimally between 2015 and 2019 (0.82 to 0.77 per 100.000 inhabitants). ${ }^{2}$ In the last 10 years, Colombia has had an average of 400 new cases per year (366 in 2019), of which 7\% correspond to children, showing that disease transmission is active. ${ }^{2,3}$

Detection of neuropathy in its early stages is not straightforward, often requiring significant clinical experience. Neurological alterations become clinically detectable only after fiber destruction has progressed past $30 \%$, and they vary significantly between leprosy types.-6 Overall, patients with high cellular reactivity (hyperergia) have a tuberculoid (TL) or borderline tuberculoid (BT) presentation and display faster and greater neuropathic compromise compared to multibacillary forms. The most frequent neurological manifestations are peripheral mononeuropathy (involvement of a single nerve trunk) which occurs mainly in the tuberculoid pole. Asymmetric multiple mononeuropathy (affection of two or more peripheral nerve trunks), appears mainly in dimorphic, lepromatous states and leprotic reactions. The ulnar, common peroneal, radial, posterior tibial, median and supraorbital nerves are the main nerves involved. $^{7-11}$

The spectrum of leprosy most associated with physical deformities is borderline leprosy, which leads to abrupt changes in the immune system that lead to a rapid involvement of various nerve trunks. Periodic clinical evaluation is essential in these patients, as well as the assessment of the level of disability at the time of diagnosis, which is carried out based on a standardized classification by the WHO. ${ }^{12}$

Descriptions of the clinical presentation of neuropathy in leprosy are scarce and often show large variations between countries. No such descriptions are available in our country and neuropathy is often overlooked by clinicians who do not know what manifestations are most common. The aim of this study was to describe the clinical manifestations of neuropathy in patients with Hansen's disease who were treated at a national referral center for leprosy in Bogotá D.C., Colombia.
Correspondence: María Alejandra PalaciosAriza, Research Unit, Fundación Universitaria Sanitas, Cl. $170 \quad \# 8-41$, Bogotá, Cundinamarca, Colombia.

E-mail: mapalaciosar@unisanitas.edu.co

Key words: Leprosy, Physical examination, Peripheral nervous system diseases, Physical disability, Epidemiologic studies.

Contributions: The authors contributed equally.

Conflict of interest: The authors declare no potential conflict of interest.

Funding: None.

Ethics and declaration of patient consent: This project was approved by the Institutional Review Board of the Hospital Universitario Centro Dermatológico Federico Lleras Acosta (Approval number 202002030037743). The authors certify that they have obtained all appropriate patient consent forms. In the form the patients have given their consent for their images. The patients understand that their names and initials will not be published, and due efforts will be made to conceal their identity, but anonymity cannot be guaranteed.

Please cite this article as: Trujillo-Ramirez L, Palacios-Ariza MA, Pradilla I, Gamboa LA. Peripheral neuropathy in leprosy: Clinical manifestations and disability in a Colombian national referral center. Dermatol Rep 2021;14:9308

Received for publication: 7 July 2021 Accepted for publication: 8 July 2021.

This work is licensed under a Creative Commons Attribution-NonCommercial 4.0 International License (CC BY-NC 4.0).

CCopyright: the Author(s), 2021

Licensee PAGEPress, Italy

Dermatology Reports 2022; 14:9308

doi:10.4081/dr.2021.9308

Publisher's note: All claims expressed in this article are solely those of the authors and do not necessarily represent those of their affiliated organizations, or those of the publisher, the editors and the reviewers. Any product that may be evaluated in this article or claim that may be made by its manufacturer is not guaranteed or endorsed by the publisher.

\section{Materials and methods}

\section{Setting and data sources}

This descriptive study retrospectively collected data from electronic health records (HER) of patients from the national referral center for the treatment of leprosy 
between January 2003 and December 2018 in Bogotá D.C., Colombia. We selected EHRs of patients with a diagnosis of leprosy confirmed by a positive bacilloscopic or pathology report. We extracted data on patient demographics, clinical characteristics of the lesions, including location and type, and features of a neurological examination.

We manually analyzed each EHR to determine the validity of the diagnosis. We excluded records with invalid diagnoses and those of patients who had not reported grade of disability. Quality of data input was verified at increments of $15 \%$ of the sample initially identified. No pre-specified sample size was defined, and all records were considered for data extraction.

\section{Study variables}

We extracted data on the dates of onset, diagnosis, sex, place of origin, type of lesion, affected skin areas, and type of leprosy. We also extracted data on the results of an exhaustive neurological examination aimed at the detection of peripheral neuropathy. Disability grades (eye, hand, foot and global) were collected as well. Treatment is reported as multi or paucibacillary. Multibacillary treatment consisted of rifampin, clofazimine and dapsone, while paucibacillary treatment consisted of rifampin and dapsone only.

\section{Statistical analysis}

We present measures of central tendency and dispersion as appropriate for the underlying distribution of continuous variables. Qualitative variables are presented as absolute and relative frequencies. Type and lesion location frequencies are summarized in UpSet plots. ${ }^{13}$ All analyses were carried out using R v.4.0.2. ${ }^{14}$

\section{Results}

The diagnostic code search produced 327 eligible EHRs. We discarded 45 records due to invalid diagnoses (misdiagnosis), lack of a report of degree of disability, or missing bacilloscopy or pathology results. Subjects were also excluded if they had negative results in both the bacilloscopy and the pathology report. The final sample consisted of 282 subjects, 48 of which had a normal neurological examination.

Our sample consisted of slightly more males than females, with a median age at diagnosis of 54 years. The patients' place of residence was most commonly urban, though slightly under $19 \%$ resided in rural communities, and most were first time con- sults. Most subjects were ultimately diagnosed as having lepromatous leprosy, with borderline tuberculous leprosy being the next most common diagnosis. Pure neuritic leprosy was the least common diagnosis, present in only two subjects. The most common lepromatous reaction was type I, and only one patient had features of both type I and II reactions. A significant number of patients had received no treatment before their consult at the referral center (Table 1).

Pain over the median nerve trunk was the most common manifestation of disease followed by pain in the radial nerve trunk. Thickening was an overall rare presentation, reaching a maximum frequency of $6.03 \%$ for the sciatic-popliteal nerve trunk (Table 2). It was more common to find both pain and thickening than thickening alone. Very few patients $(3.55 \%)$ displayed pain and/or thickening of the auricular nerve trunk. The pattern of concurrent thickening of the radial, median, and ulnar nerves was most common $(n=26)$.

Only five subjects presented with no skin lesions. Slightly over $80 \%$ of patients presented with plaques, and it was more common for patients to present with plaques only. The next most common presentation was that of both plaques and macules. Ulcers were the least common type of lesion, present in only $4.96 \%$ of subjects (Figure 1A).

Overall, sensitive alterations were more common than motor ones. The posterior tibial nerve was affected in the most patients, followed by the anterior tibial nerve. However, the ulnar nerve concentrated the greatest number of patients with both motor and sensitive impairments (Table 3 ).

Lesions found in the abdomen, thorax, pelvis or back were grouped into the Trunk category. The lower limbs were affected in $75.2 \%$ of subjects, being the most common-
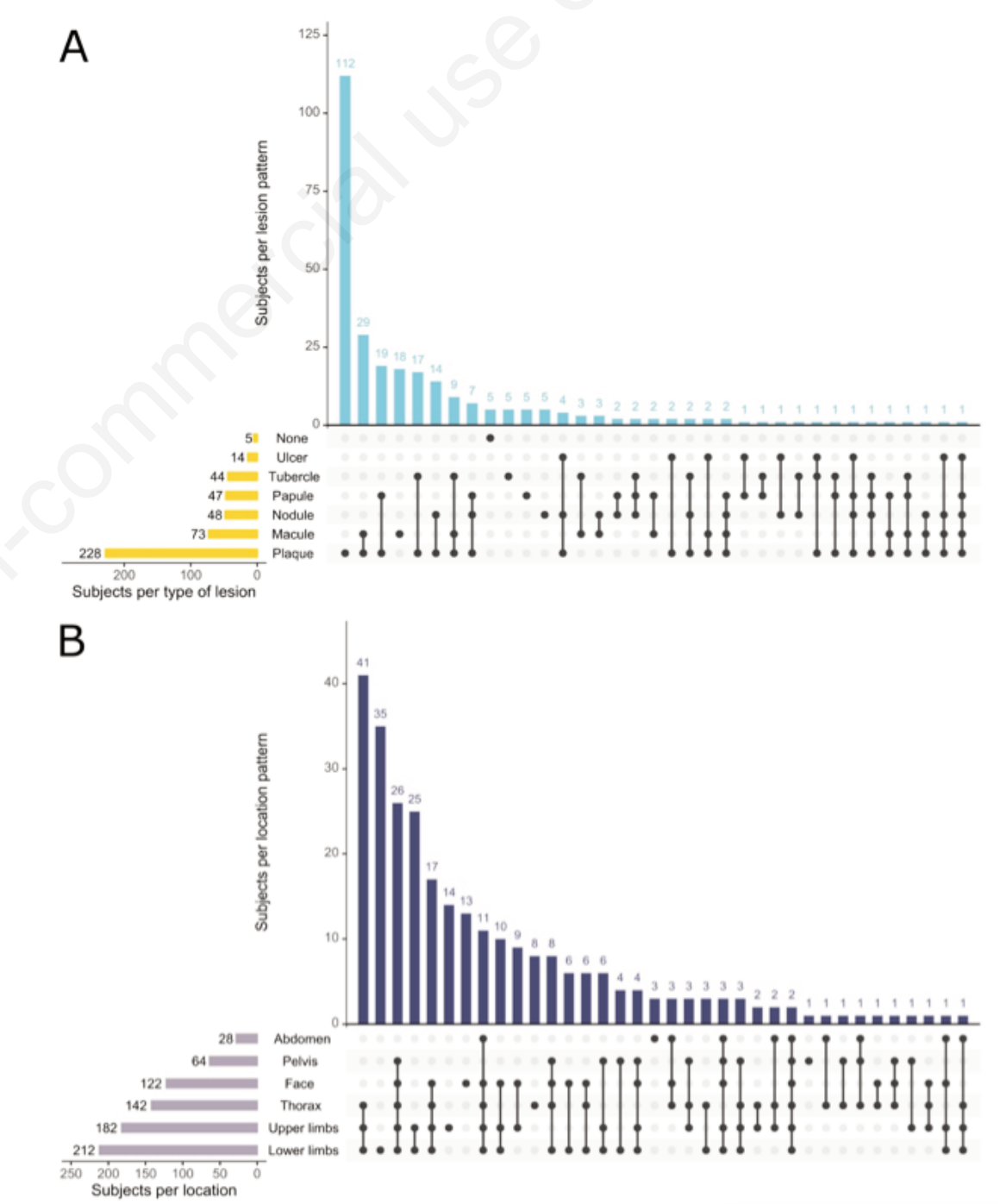

Figure 1. Lesion Characterization A. Type B. Location. 
ly affected region. The face was the least compromised area with $43.3 \%$ of the cases. Most patients presented with lesions distributed over all body regions considered $(22.7 \%)$, the second most common pattern including all regions but the face $(18.09 \%)$ (Figure 1B).

Less than $15 \%$ of our subjects had a WHO degree of disability (Table 4) of 1 in individual body parts. ${ }^{12}$ Likewise, less than $10 \%$ were graded as 2 in the body parts considered. Manifestations of grade 2 disability included the benediction sign, the absence of the uvula, and finger and nail dystrophy (Figure 2). The eyes were least likely to be compromised. Globally, $23.8 \%$ of our patients had some degree of disability.

\section{Discussion}

Given the considerable morbidity that

Table 1. Clinical and epidemiologic characteristics of 282 study participants.

\begin{tabular}{lc}
\hline Variable & N $(\%)$ \\
Male Sex & $162(57,45)$ \\
Marital status & \\
Single & $143(50,71)$ \\
Common-law marriage & $38(13,48)$ \\
Marriage & $69(24,47)$ \\
Divorced & $19(6,74)$ \\
Widowed & $13(4,61)$ \\
Urban residents & $229(81,21)$ \\
\hline Primary complaint & \\
First time consult & $229(81,21)$ \\
Relapse & $53(18,79)$ \\
Positive Bacilloscopy & $174(61.7)$ \\
Bacillary Index & \\
Negative & $107(37.94)$ \\
Paucibacillary & $6(2.13)$ \\
Multibacillary & $169(59.93)$ \\
(+) Pathology Report & $281(99.65)$ \\
\hline Leprosy type & \\
Tuberculoid & $20(7.09)$ \\
Borderline tuberculoid & $54(19.15)$ \\
Borderline & $36(12.77)$ \\
Borderline lepromatous & $47(16.67)$ \\
Lepromatous & $112(39.72)$ \\
Pure neuritic & $2(0.71)$ \\
Indeterminate & $11(3.9)$ \\
Lepromatous Reaction & \\
Type I & \\
Type II & $62(21,99)$ \\
Both & $26(9,22)$ \\
None & $1(0,35)$ \\
\hline Prior Treatment & $193(68,44)$ \\
Multibacillary & \\
Paucibacillary & \\
Other & \\
No treatment & $9(3,12,19)$ \\
Age at diagnosis, Median (IQR) & \\
Time since first skin lesion (years) & $1(2)$ \\
\hline & \\
& \\
\hline
\end{tabular}

its complications produce, leprosy continues to generate a significant psychosocial and economic impact. Although eradication has been possible in other countries, it remains a challenging public health issue in Colombia.

The largest case series of neuropathy in leprosy have been reported by groups in India and Brazil, countries that share some social risk factors for the disease with Colombia, including poverty, overcrowding, malnutrition and limited access to health care. ${ }^{1,15}$ Most of our sample resided in cities, which are expected to offer better living conditions than the countryside in Colombia. However, this does not guarantee that the communities our patients come from are free from risk factors for transmission. Future studies could concentrate on the living conditions of incident cases.

The WHO has shown that people with leprosy are predominantly male, a consis- tent finding as shown in our sample, in another study carried out at our referral center, in national epidemiological reports and in international studies..$^{1,3,15-19}$ Disability is also more frequent in males, as was the case in our study. Some authors have hypothesized that this sex difference might be the result of socioeconomic factors. Male patients may delay care due to long work hours and income instability, which limit opportunities for appointments. The overrepresentation of males in physical labor jobs could have a dual effect. Some activities in physical labor may predispose patients to deformity, and the social stigma of the disease may limit work opportunities. ${ }^{15,20,21}$

Patients over 55 years were the most commonly affected age group, which is consistent with both national and international studies, and which may reflect the chronicity of the disease and the long and

Table 2. Disease manifestations in nerve trunks.

\begin{tabular}{lcccc} 
Nerve & \multicolumn{1}{c}{ None } & Pain & Thickening & Both \\
Auricular & $272(96.45)$ & $2(0.71)$ & $2(0.71)$ & $6(2.13)$ \\
Ulnar & $197(69.86)$ & $57(20.21)$ & $4(1.42)$ & $24(8.51)$ \\
\hline Median & $167(59.22)$ & $79(28.01)$ & $8(2.84)$ & $28(9.93)$ \\
Radial & $184(65.25)$ & $62(21.99)$ & $12(4.26)$ & $24(8.51)$ \\
\hline Sciatic-popliteal & $243(86.17)$ & $16(5.67)$ & $17(6.03)$ & $6(2.13)$ \\
Posterior tibial & $246(87.23)$ & $13(4.61)$ & $14(4.96)$ & $9(3.19)$ \\
\hline Sural & $265(93.97)$ & $7(2.48)$ & $6(2.13)$ & $4(1.42)$ \\
\hline
\end{tabular}

Table 3. Type of impairment by examined nerve.

\begin{tabular}{lcccc} 
Nerve/Region & None & \multicolumn{2}{c}{$\begin{array}{c}\text { Type of impairment } \\
\text { Sensitive }\end{array}$} & $\begin{array}{c}\text { Motor } \\
\text { Face (Trigeminal/Facial) }\end{array}$ \\
Radial & $237(84.04)$ & $29(10.28)$ & $13(4.61)$ & $3(1.06)$ \\
\hline Ulnar & $198(70.21)$ & $65(23.05)$ & $4(1.42)$ & $15(5.32)$ \\
Median & $155(54.96)$ & $71(25.18)$ & $17(6.03)$ & $39(13.83)$ \\
Anterior tibial & $195(69.15)$ & $60(21.28)$ & $15(5.32)$ & $12(4.26)$ \\
Posterior tibial & $183(64.89)$ & $73(25.89)$ & $6(2.13)$ & $20(7.09)$ \\
\hline
\end{tabular}

Table 4. Degree of disability.

\begin{tabular}{lccc} 
Body Part & 0 & 1 & 2 \\
Right Hand & $257(91.13)$ & $14(4.96)$ & $11(3.9)$ \\
Left Hand & $253(89.72)$ & $11(3.9)$ & $18(6.38)$ \\
\hline Right Foot & $236(83.69)$ & $34(12.06)$ & $12(4.26)$ \\
Left Foot & $233(82.62)$ & $42(14.89)$ & $7(2.48)$ \\
\hline Right Eye & $275(97.52)$ & $4(1.42)$ & $3(1.06)$ \\
Left Eye & $275(97.52)$ & $2(0.71)$ & $5(1.77)$ \\
\hline Global Disability & $215(76.24)$ & $35(12.41)$ & $32(11.35)$ \\
\hline
\end{tabular}


variable incubation times. ${ }^{3,16,19,22,23}$ Two subjects below the age of 15 were identified in this study, one of whom displayed grade 2 disability. Different public policies on leprosy eradication, both global and national, have key goals of no new cases in children and no cases with visible deformity, so these two cases point to deficiencies in our public health system. ${ }^{21}$

Most of our patients had a diagnosis of multibacillary leprosy or borderline forms, both of which are more prone to neuropathic manifestations, as shown by Moschioni et al. ${ }^{15}$ Leprotic reactions are key risk factors which increase risk by up to $96 \%$ (type I most common in our study). Other key factors are the involvement of three or more nerve trunks, greater time since disease onset ( $>1$ year) and multibacillary states. ${ }^{16,24}$ This would explain the disability distribution in our patients, most of whom were diagnosed after at least one year from symptom onset. In other reports, $27.7 \%$ of patients had grade 1 disability and $12.1 \%$ to grade $2.2,3$

Other retrospective observational studies have shown that neuropathy may arise at any moment, regardless of treatment. ${ }^{15,19,20,24}$ Although early treatment reduces the frequency of neuropathy in groups of patients, this does not mean that vigilance must be relaxed over time in individual patients. Remaining vigilant may help prevent severe complications. For example, our study showed that the anterior and posterior tibial nerves were most commonly affected, the latter being a risk factor for complications related to plantar anesthesia like ulcers, deformity and even bone resorption. ${ }^{8,23}$

Unlike previous reports, neural thickening was not a frequent finding in our population. Between $75-94 \%$ of cases in a cohort of 303 patients with lepromatous leprosy

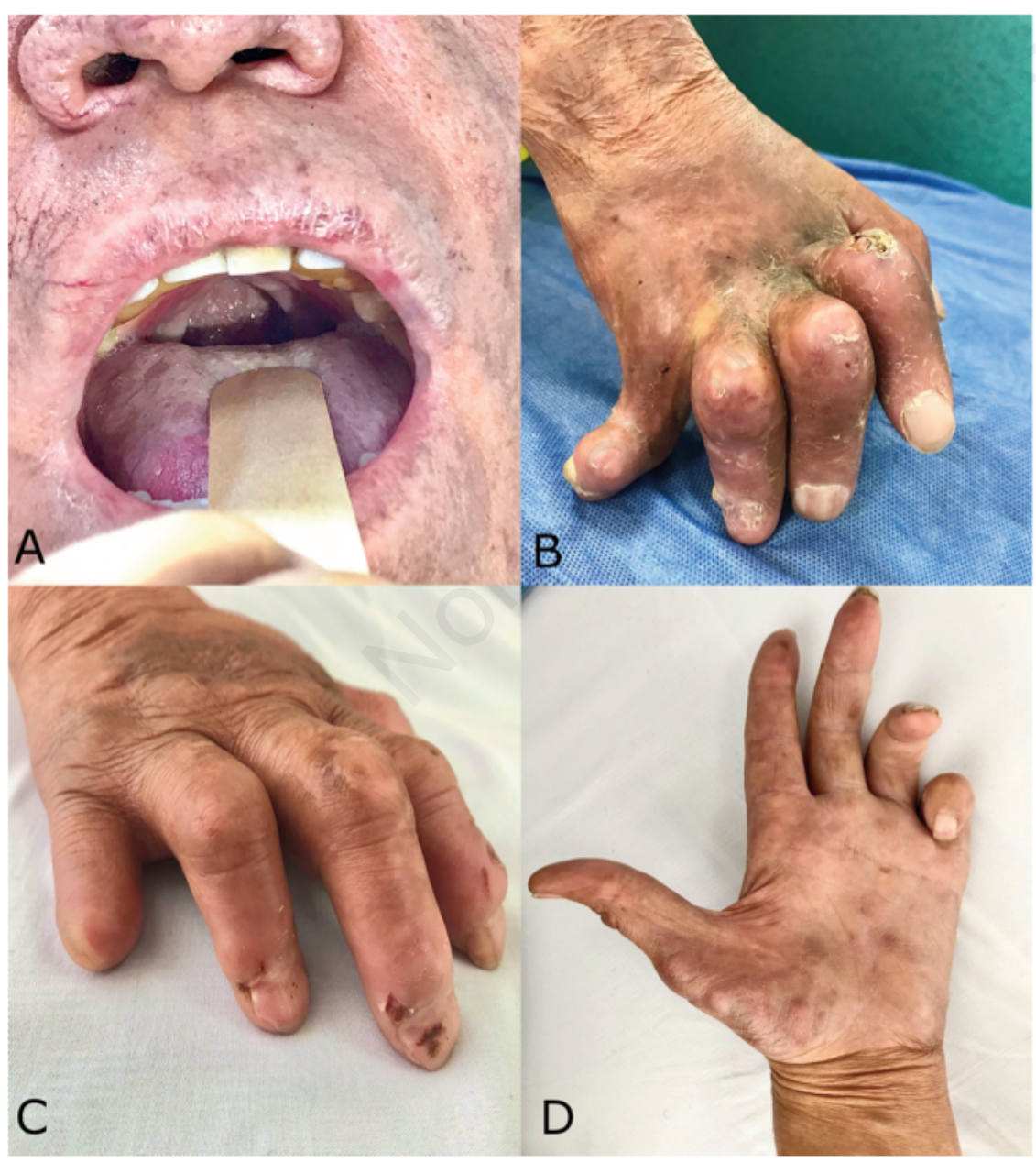

Figure 2. Patients with lepromatous leprosy and grade 2 disability. $1^{\text {st }}$ Patient: Absence of the uvula and nasal infiltration (a). Finger and nail dystrophy secondary to bone resorption (b). $2^{\text {nd }}$ Patient: Claw hand, bone resorption and traumatic erosions (c). 3rd Patient: Benediction sign (d). displayed this alteration in a multi-center study carried out in India. ${ }^{25}$ However, that cohort differed significantly from our sample with regards to reported risk factors for nerve thickening. The Indian cohort included significantly more males $(72.6 \%)$, had patients with greater times since disease onset ( $38.2 \%$ over 12 months), and a greater degree of disability $(50.5 \%)$. Variations in the frequency of this finding may also be explained by the subjectiveness of the examination, in which the physician palpates the compromised nerve trunks. It has been shown that agreement between physical examination by experienced personnel and nerve ultrasonography is of only $0.3 .^{26}$ Imaging has the advantage of being able to evaluate a greater portion of the nerve, including portions that lie deep to the skin, where palpation cannot identify any changes. Although the clinical utility of ultrasonography in the management of leprosy is yet to be shown, it appears that efforts to educate physicians in nerve palpation may be fruitless.

The evaluation of the degree of disability was one of the key objectives for this study since this represents an early diagnostic marker of leprosy. Early detection of peripheral neuropathy is complex, mainly because it is highly dependent on the physician's experience. In the early stages of the disease itself, clinical manifestations of neural impairment might be imperceptible. Both the clinical examination and the interpretation of diagnostic testing depend on clinical expertise to make them reliable. Additionally, healthcare workers in Colombia show a lack of knowledge of this disease. This was shown by Gómez et al. in a study on 249 patients, up to $70 \%$ of which had initially been misdiagnosed and mistreated. That study also found that the average delay from disease onset to diagnosis was of 33.5 months, and that $14.5 \%$ of the subjects had grade 2 disability at diagnosis. ${ }^{27}$ This might account for the high frequency of neurological alterations and disability in the Colombian population.

\section{Conclusions}

In this study, the age and leprosy type distributions in patients with neuropathy were consistent with reports from other countries. The anterior tibial, ulnar and posterior tibial nerves were most commonly affected which is also consistent with prior reports. However, nerve trunk thickening was much more infrequent, which might be a consequence of subjectiveness in the examination and differences in sex distribu- 
tion, degree of disability and time since disease onset. The frequency of morbidity and disability found in this sample, though low when compared with other series, fails to meet public health goals, including those limiting disability in younger subjects.

\section{References}

1. World Health Organization. Global Leprosy (Hansen Disease) Update, 2019: Time to Step-up Prevention Initiatives. World Health Organization; 2020:417-440. Accessed December 7, 2020. https://apps.who.int/iris/ handle/10665/334141

2. Instituto Nacional de Salud. Lepra en Colombia 2019.; 2020:30. Accessed December 7, 2020. https://www. ins.gov.co/buscador-eventos/ BoletinEpidemiologico/2020_Boletin_ epidemiologico_semana_3.pdf

3. Fadul Pérez SE. Lepra Periodo IV, Colombia, 2019. Instituto Nacional de Salud; 2019:16. https://www.ins.gov. co/buscador-eventos/Informes deevento/LEPRA\%20SEMESTRE\%20I\%202 019.pdf

4. Penna MLF, Penna GO, Iglesias PC, et al. Anti-PGL-1 Positivity as a Risk Marker for the Development of Leprosy among Contacts of Leprosy Cases: Systematic Review and Meta-analysis. PLoS Negl Trop Dis 2016;10: e0004703.

5. Kumar V, Sachan T, Natrajan M, Sharma A. High resolution structural changes of Schwann cell and endothelial cells in peripheral nerves across leprosy spectrum. Ultrastruct Pathol 2014;38:86-92.

6. Pandhi D, Chhabra N. New insights in the pathogenesis of type 1 and type 2 lepra reaction. Indian J Dermatol Venereol Leprol 2013;79:739-49.

7. Eichelmann K, González González SE, Salas-Alanis JC, Ocampo-Candiani J. Leprosy. An update: definition, pathogenesis, classification, diagnosis, and treatment. Actas Dermosifiliogr

\section{3;104:554-563.}

8. Nascimento OJM. Leprosy neuropathy: clinical presentations. Arq Neuropsiquiatr 2013;71:661-6.

9. Lau KHV. Neurological Complications of Leprosy. Semin Neurol 2019;39:462-71.

10. Berkowitz AL, Raibagkar P, Pritt BS, Mateen FJ. Neurologic manifestations of the neglected tropical diseases. J Neurol Sci 2015;34920-32.

11. Sindic CJM. Infectious neuropathies. Curr Opin Neurol 2013;26:510-5.

12. Brandsma JW, Van Brakel WH. WHO disability grading: operational definitions. Lepr Rev 2003;74:366-73.

13. Gehlenborg N. UpSetR: A More Scalable Alternative to Venn and Euler Diagrams for Visualizing Intersecting Sets; 2019. https://CRAN.Rproject.org $/$ package $=$ UpSetR

14. R Core Team. R: A Language and Environment for Statistical Computing. $\mathrm{R}$ Foundation for Statistical Computing; 2020. https://www.R-project.org/

15. Moschioni C, Antunes CM de F, Grossi MAF, Lambertucci JR. Risk factors for physical disability at diagnosis of 19,283 new cases of leprosy. Rev Soc Bras Med Trop 2010;43:19-22.

16. Guerrero MI, Muvdi S, León CI. Retraso en el diagnóstico de lepra como factor pronóstico de discapacidad en una cohorte de pacientes en Colombia, 2000 - 2010. Rev Panam Salud Pública 2013;33:137-43.

17. Aben-Athar CYUP, Lima SS, Ishak R, Vallinoto ACR. Assessment of the sensory and physical limitations imposed by leprosy in a Brazilian Amazon Population. Rev Soc Bras Med Trop 2017;50:223-8.

18. Bandeira SS, Pires CA, Quaresma JAS. Nerve Damage in Young Patients with Leprosy Diagnosed in an Endemic Area of the Brazilian Amazon: A CrossSectional Study. J Pediatr 2017;185:143-8.

19. Shumet T, Demissie M, Bekele Y. Prevalence of Disability and Associated Factors among Registered Leprosy
Patients in All Africa Tb and Leprosy Rehabilitation and Training Centre (ALERT), Addis Ababa, Ethiopia. Ethiop J Health Sci 2015;25:313-20.

20. de Paula HL, de Souza CDF, Silva SR, et al. Risk Factors for Physical Disability in Patients With Leprosy: A Systematic Review and Meta-analysis. JAMA Dermatol 2019;155:1120-8.

21. World Health Organization. Global Leprosy Strategy 2016-2020. Accelerating towards a Leprosy-Free World. Monitoring and Evaluation Guide. 1ra ed. Regional Office for South-East Asia, World Health Organization; 2017. Accessed November 23, 2020. https://apps.who.int/iris/handle/10665/2 54907

22. Santos VS, de Matos AMS, de Oliveira LSA, et al. Clinical variables associated with disability in leprosy cases in northeast Brazil. J Infect Dev Ctries 2015;9:232-8.

23. Sarker UK, Uddin MJ, Chowdhury R, et al. Clinical Profile of Peripheral Neuropathy in Leprosy. Mymensingh Med J MMJ 2015;24:671-8.

24. Dos Santos AR, Silva PR de S, Steinmann P, Ignotti E. Disability progression among leprosy patients released from treatment: a survival analysis. Infect Dis Poverty 2020;9:53.

25. van Brakel WH, Nicholls PG, Das L, et al. The INFIR Cohort Study: investigating prediction, detection and pathogenesis of neuropathy and reactions in leprosy. Methods and baseline results of a cohort of multibacillary leprosy patients in north India. Lepr Rev 2005;76:14-34.

26. Jain S, Visser LH, Praveen TLN, et al. High-Resolution Sonography: A New Technique to Detect Nerve Damage in Leprosy. PLoS Negl Trop Dis 2009;3:e498.

27. Gómez L, Rivera A, Vidal Y, et al. Factors associated with the delay of diagnosis of leprosy in north-eastern Colombia: a quantitative analysis. Trop Med Int Health 2018;23:193-8. 